ÉGYPTE

monde arabe

\section{Égypte/Monde arabe}

14 | 1993

Dits et écrits, mémoires et rites

\title{
Contes et conteurs de Louqsor
}

\section{Rosine Schautz}

\section{(2) OpenEdition}

\section{Journals}

Édition électronique

URL : https://journals.openedition.org/ema/551

DOI : 10.4000/ema.551

ISSN : 2090-7273

\section{Éditeur}

CEDEJ - Centre d'études et de documentation économiques juridiques et sociales

\section{Édition imprimée}

Date de publication : 30 juin 1993

Pagination : 15-36

ISSN : 1110-5097

\section{Référence électronique}

Rosine Schautz, "Contes et conteurs de Louqsor », Égypte/Monde arabe [En ligne], 14| 1993, mis en ligne le 08 juillet 2008, consulté le 07 juillet 2022. URL : http://journals.openedition.org/ema/551 ; DOI : https://doi.org/10.4000/ema.551

Ce document a été généré automatiquement le 7 juillet 2022.

Tous droits réservés 


\title{
Contes et conteurs de Louqsor
}

\author{
Rosine Schautz
}

Pour le professeur Werner VycichI

« Raconte une belle histoire ou je te tue... »

A. Khatibi, Ombres japonaises, Fata Morgana, 1988.

1 Cette étude s'articule autour de trois axes. En premier lieu, je me propose d'«aller voir » du côté des conteurs égyptiens en quoi ils restent, aujourd'hui encore, les dépositaires d'une parole qui constitue la mémoire orale. J'analyse également le contexte et les règles selon lesquelles ils pratiquent leur art. .

2 J'ai choisi la région de Louqsor parce qu'elle est située à un carrefour de la HauteÉgypte. De par sa situation géographique tout d'abord: le désert, si important dans la poésie arabe, est là, sous les yeux. De par sa situation religieuse ensuite: les deux religions monothéistes, chrétienne et musulmane, cohabitent harmonieusement sur les vestiges des temples et tombeaux pharaoniques. De par la richesse de son histoire, enfin, que l'on peut, par couches successives, redécouvrir dans les contes.

3 Les contes proprement dits sont traités en seconde partie. Il me semblait intéressant de voir, dans un premier temps, en quoi ces contes sans cesse reinventés disent, au moyen d'une langue arabe dialectale très riche, la permanence d'une tradition orale. Et, dans un deuxième temps, comment ils sont en fait le miroir dans lequel se reflète la vie quotidienne égyptienne, élément étroitement lié à la question de l'identité.

4 Enfin, en dernière partie, j'évoquerai l'avenir incertain des contes et des conteurs dans leur expression et leur pratique traditionnelle. Car s'ils restent encore présents lors des fêtes religieuses et populaires à dates fixes, ils n'ont en revanche aucune chance d'investir de nouveaux espaces de la vie sociale et culturelle actuelle.

Conteurs

5 On a longtemps cru que les traditions orales étaient destinées à mourir un jour ou l'autre, parce que la restitution par écrit des contes et des légendes dénaturerait leurs messages et leurs enseignements qui prennent racine dans l'histoire mythique et dans ce que d'aucuns appellent la sagesse " populaire».

6 Or, même après les nombreuses tentatives de transcription de ce patrimoine culturel, la région de Louqsor a su conserver jusqu'à aujourd'hui une tradition orale que l'on 
pourrait qualifier de populaire. Est-ce parce qu'un des grands poètes contemporains de Haute-Égypte, 'Abd al-Rahmân al-Abnûdi, originaire de Qena $(60 \mathrm{~km}$ au nord de Louqsor), s'est attelé à la tâche ambiguë de collecter et d'enregistrer les chapitres d'une des gestes arabes les plus fameuses'? Est-ce parce que le festival du saint patron de Louqsor (mawlîd Abû-l-Hajjâj) continue d'avoir lieu, année après année, ainsi que les nombreuses fêtes et foires en l'honneur des cheikhs locaux? Est-ce parce que le tourisme, extrêmement développé dans cette région, joue a contrario un rôle de gardefou de la tradition? Ou parce que le folklore est devenu un produit commercialisable au même titre que les sites archéologiques?

7 La réponse ne saurait être définitive, non seulement parce qu'il s'agit de tout cela à la fois, mais aussi parce qu'on ne peut parler d'une tradition orale, mais bien plutôt d'un ensemble de phénomènes liés, de près ou de loin, à l'oralité.

Louqsor hier et demain

8 La région de Louqsor est située de part et d'autre du Nil, et jouit de ce fait d'un statut étrange. Scindée géographiquement, elle perpétue les animosités séculaires entre les habitants de l'est et ceux de l'ouest. On retrouve d'ailleurs ces inimitiés jusque dans les proverbes : Elle jâi mel-gharb ma yesurresh el-galb («Ce qui vient de l'ouest ne rend pas le cœur heureux»). Mais Louqsor est aussi un lieu de rencontre entre coptes et musulmans qui se partagent les gloires du passé. Cela mérite d'être remarqué à l'heure où l'acceptation des différences devient chaque jour plus contraignante. Enfin, c'est une région où le tourisme est exacerbé, surdéveloppé, notamment depuis les médiatiques représentations d'Aïda au cœur du temple de Louqsor, ou encore depuis que la ville s'est dotée d'un aéroport international.

9 Pourtant, malgré tous ces éléments et probablement justement à cause d'eux, la région demeure profondément ancrée dans ses traditions, notamment agricoles, comme c'est le cas des provinces voisines, le long de la Vallée du Nil. On y cultive la canne à sucre (gasab), le blé (gamh), le trèfle (barsîm), les fèves (fûl), on y vit au rythme des récoltes et selon les saisons, comme le veulent les différents calendriers. Et il est tout à fait intéressant d'y prêter attention : car souvent les conteurs sont, de jour, les personnages bien vivants de cette vie qui s'écoule dans les champs, loin de la ville et d'une certaine excitation urbaine. Et également parce que c'est de cela que parient les conteurs. Cette quotidienneté empiète sur les contes et les rend, dès lors, doublement "parlants ». En outre, la singularité géographique de Louqsor est toujours présente, implicitement ou explicitement, dans les histoires racontées, qu'elles soient coptes ou arabes, chrétiennes ou musulmanes. J'y reviendrai.

C'est donc bien parce que cette région est connue presqu'uniquement pour ses ruines et ses sites archéologiques qu'il fallait y chercher des itinéraires différents: ceux qui mènent à travers les temps aux sources mêmes de la littérature arabe en général, de la poésie dialectale en particulier.

Devenir conteur

11 Etre conteur ou poète traditionnel (châ'ir) résulte d'une des trois situations suivantes, voire de la superposition de certaines d'entre elles : on accède à la "profession " lorsqu'on n'a plus que soi-même pour survivre, c'est-à-dire lorsque l'on n'a ni terres à cultiver, ni parenté à nourrir ou famille à soutenir ; ou parce que l'on peut mémoriser sans peine des centaines de couplets (adwâr) ou de poèmes en dialectal (mawawîl) après incitation d'un proche ou sur invitation d'un maitre ; et, bien sûr, parce que l'on côtoie depuis son plus jeune âge conteurs, poètes et musiciens. 

de la région de Louqsor et d'Assouan, Jamâl ne peut quant à lui être considéré comme conteur. Il serait plutôt un intermédiaire ou un passeur ${ }^{2}$, quelqu'un qui peut décrypter et expliquer cette poésie dialectale sa'îdi et la répéter, sans avoir toutefois la possibilité d'inventer des vers qui «sonnent» et qui «disent». Je reviendrai plus bas sur ces notions. C'est en partie grâce à Jamâl que J'ai pu mesurer l'importance de la poésie traditionnelle dialectale dans la vie de cette région. Je tenais à l'inclure dans ces différents portraits, parce que n'est pas poète-conteur qui veut ou qui peut. Il ne faut pas oublier que la profession de conteur est encore et toujours, comme c'est le cas par ailleurs dans nombre de pays, tout à fait déconsidérée, voire méprisée et malfamée. Comme le dit le proverbe sa'îdi : Elle yedrab al-târ ma 'andush 'asîl («Celui qui frappe le tambourin n'a pas de souche »). On assimile très souvent les conteurs aux vagabonds et aux mendiants, voire aux fous ${ }^{3}$ et bien sûr aux Tsiganes, qui pourtant ne participent guère à la tradition des conteurs. Tsigane qui joue de la rabâba (le violon à deux cordes de Haute-Égypte), comme le notait aussi G. Canova'. En outre, on aime à croire dans la région qu'ils font partie d'une tariqa (confrérie soufie), mais si cela est parfois vrai, il doit s'agir le plus souvent d'une réputation que leur font ceux qui ne sont familiers ni du monde des conteurs ni de celui des confréries. Il reste que ces dernières sont très présentes à Louqsor et dans les villages voisins, ainsi que dans ces mêmes foires qui sont les points de rencontre des poètes, des Tsiganes et des musiciens. Ceci explique peut-être que l'on finisse par prendre les uns pour les autres.

Châ'ir, râwi, hakawi

Si l'on demande à Jamâl ce qu'est un conteur et, partant de là, pourquoi il n'en est pas un, il ne peut que répondre qu'Awadallah ou Rizg le sont parce qu'ils sont " poètes » (chu'ara'). On s'aperçoit alors qu'il y a plusieurs degrés dans l'art de conter, et qu'être poète (châ'ir) en serait le summum (daraja ûla). Suivrait le conteur (râwi) qui ne chante pas, mais parle (byetkallem, ma beyghannîch), et enfin celui qui raconte une histoire (hakawi), n'importe qui (ayye hadd) pouvant être hakawi pour autant qu'il commence son histoire par une formule comme «il était une fois » (kân ya makân) $)^{5}$.

Les conteurs et poètes sont la plupart du temps accompagnés de musiciens qui frappent le rythme sur le tambourin (târ) ou qui soutiennent et développent la mélodie du conte au hautbois (zammâra ou mizmâr baladi ou encore abâ). Ce dernier est l'instrument traditionnel des conteurs de la geste hilalienne, qui chantent généralement d'une voix nasillarde pour faire écho au son grinçant du hautbois. La flûte (nay) n'est utilisée que très exceptionnellement lors de ces récitations: étant réservée uniquement aux épisodes plus intimes, elle accompagne par exemple les chansons d'amour ou les poèmes improvisés en langue dialectale (mawawil) qui parsèment le conte. On peut d'ailleurs aussi être amené à entendre un conte ou un épisode de la geste ou de la vie 
d'un saint sans autre accompagnement que celui qu'exécute le conteur de ses propres mains.

Conter au fil des saisons

17 Au départ, les poètes sont là pour divertir, dans les champs ou lors des veillées de travail agricole, et reçoivent en salaire les fruits des premières récoltes. Avant l'achèvement du Haut-Barrage d'Assouan dans les années 66-67, le Nil, en raison de ses crues régulières, donnait un rythme à la vie paysanne de la Vallée. Il y avait trois saisons annuelles : lors de la crue elle-même (nîli), en hiver (chitwi) et en été (sêfi). Ces saisons procuraient des occasions pour le conte et la récitation. A celles-ci s'ajoutaient les nombreuses fêtes des calendriers copte et musulman; les longues nuits entre amis pendant le mois de Ramadan, les soirées lors du festival d'Abû-l-Hajjâj et les fêtes privées organisées par des particuliers à l'occasion des fiançailles, des mariages ou des baptêmes.

18 Aujourd'hui, les survivants de cette époque révolue appartiennent à une autre génération - ils ont tous, ou presque, plus de 80 ans - ce qui ne les empêche pas de garder en tête des milliers de vers et quantité d'histoires.

Un auditoire qui sait

19 Si l'on admet que les conteurs se font de plus en plus rares du fait de la transformation des modes de vie, on est en droit de se demander si les auditeurs ne font pas eux aussi défaut. Les conteurs, en effet, ne se produisent que dans certaines circonstances (fêtes et foires) mais aussi devant un certain public. Une geste ne se récite pas devant n'importe quel auditoire, comme le montrent nombre d'études récentes sur la tradition orale. Premièrement parce que la parole est en quelque sorte "sacrée ", héritée de la bouche des ancêtres, et ne peut dès lors être galvaudée. Deuxièmement - c'est une constante de l'oralité - parce que l'histoire, en tant que succession de faits historiques ou mythiques, ne se déroule pas selon un ordre chronologique.

20 Peut-être, dans le contexte arabe, cette caractéristique fait-elle aussi partie du rapport à la langue via le Coran, dont les sourates sont classées par ordre décroissant de longueur et non par ordre d'apparition des faits dans le temps. Livre par excellence, livre de référence dont découlent non seulement la doctrine religieuse, mais également la loi et la grammaire, ainsi que celles de la métrique de la poésie arabe, ce qui le rend indispensable à quiconque s'exprime dans cette tangue, qu'il soit musulman, juif, chrétien ou agnostique. Troisième raison du choix d'un public spécifique : l'auditeur doit savoir le conte presque par cœur, en tout cas en bien connaître la trame, pour apprécier la performance du poète. La parole ainsi offerte ne doit pas «tomber par terre ", comme le dit si bien 'Awadallah. L'étude comparative des traditions orales africaines révélerait certainement encore bien d'autres raisons. On assiste là à une sorte de paradoxe ; écouter et entendre uniquement ce que l'on sait déjà, c'est-à-dire comprendre et apprécier un moment où le conte a lieu, plutôt qu'une histoire parmi d'autres. Si l'auditoire n'acceptait pas cette règle du jeu (mais est-ce un «jeu»?), les contes et les histoires deviendraient anecdotes ou bavardage. En ce sens, le conteur se rapproche de l'acteur de théâtre de répertoire - comme on dit au Français - acteur qui est davantage un interprète - au sens où on l'entend généralement en musique - que quelqu'un qui joue un rôle ou un personnage une fois dans sa vie.

21 Ce rapport conteur-auditeur est particulièrement frappant pour qui n'est pas familier de ce type de relation. D'abord, parce que lorsqu'on vient du monde de l'écrit, on a du mal à saisir comment un conteur, à travers et malgré les règles si bien définies de l'art 
de conter, arrive encore à inventer l'histoire. C'est-à-dire non seulement à l'imaginer, mais aussi à la retrouver, nue, et à l'agrémenter de sa fantaisie à lui. Ce handicap qui est le nôtre est le fruit de notre propre rapport à l'oralité, qui passe par notre fréquentation de la poésie déclamée ou par notre goût des lectures publiques ou encore par le truchement des acteurs de théatre, qui donnent à entendre des textes mis en voix ${ }^{6}$ J'exclus à dessein toute allusion à la musique, dont je reparlerai plus bas, pour ne garder que trois exemples de "mise en scène" de la parole qui déterminent nos comportements et nos attitudes sociales à l'égard du langage.

Dans le cas qui nous intéresse ici, celui des auditeurs, il s'agit de tout autre chose. Pas seulement de la mise en scène d'une "parole donnée ", mais de quelque chose que l'on pourrait peut-être comparer, mutatis mutandis, à l'art culinaire ; on sait ce que l'on va manger lorsqu'on commande au restaurant un plat que l'on aime, mais on ne sait pas sous quelle forme exactement il nous parviendra, toute la subtilité du chef consistant à la fois à nous rassurer sur notre choix et à nous dérouter quant à la réalisation du mets. Plus le résultat sera accompli «dans les règles de l'art» tout en restant riche de mystères, et plus on sera sous le charme au sens fort. Lors d'une performance, le poète lui aussi doit émerveiller son "client » : il n'a pas le droit de le tromper sur la matière (l'épisode), il n'a pas le droit de l'égarer en mélangeant mal les phrases et les couplets, et il n'a pas le droit de fixer de nouvelles règles (du jeu, de l'art\} qui changeraient le goût général de l'épopée ou de la geste.

L'auditeur devient ainsi une sorte de conteur en puissance, et l'on peut se demander si les fonctions de conteur sont nettement définies. Cela est vrai aussi dans d'autres cultures où les littératures orales continuent de participer à l'organisation de la vie sociale, mais, dans le cas de la culture arabe, cela fait en outre penser à ce qui se passe dans le domaine de la musique traditionnelle où l'auditeur est perçu comme faisant partie du processus créateur d'émotion?.

D'autre part, le conteur qui mène sa parole et conduit son discours peut avoir recours à la répétition d'un mot, d'un vers ou d'une bribe de phrase, comme c'est le cas aussi dans les chansons, afin de créer un écho et une émotion. A l'instar de ce qui se passe dans les confréries religieuses, le conteur devient une sorte de maitre de cérémonie qui mesure l'impact émotionnel de la parole sur l'auditoire, ce qui lui permet de comprendre l'action de cette même parole sur cet auditoire et de moduler sa récitation en conséquence.

Dans une confrérie, lors des séances de mise en exercice d'une parole - qui peut être un des noms d'Allah - le maître (murchid) exerce de même manière une technique orale d'enseignement pour ses disciples (muridîn), en surveillant lui aussi l'effet de la parole sur celui qui la reçoit, la dit et la vit de l'intérieur.

L'auditoire qui sait écouter et comprendre l'histoire des Benî Hilâl (sîrat Benî Hilâl) et la poésie dont elle est construite a aujourd'hui quasiment disparu. Seuls restent quelques auditeurs qui en fredonnent ça et là les épisodes, surtout depuis leur récente apparition dans les bazars sous forme de cassette. Toutefois, dans la performance telle qu'on peut encore la voir à Louqsor, lors du mawlid ' Abû-l-Hajjâj par exemple, on assiste à un spectacle de parole mise en scène. Le découpage en épisodes, sur trois ou cinq jours, des exploits d'Abû Zêd l'Hilalien ainsi que l'utilisation d'instruments de musique traditionnels redonnent vie au rite qu'est l'art de conter. Par ailleurs, l'usage d'accessoires comme le bâton des jouteurs, et l'accompagnement de l'histoire par des 
gestes eux aussi traditionnels et obéissant à des normes très précises, font de ces soirées à la belle étoile, plus récemment sous chapiteau, un lieu de rencontres et un moment de convivialité fort appréciés quinze jours avant Ramadan.

On sait, notamment depuis les travaux de P. Zumthor et de G. Calame-Griaule, l'importance que revêtent le corps et les gestes du conteur lors de la récitation. On comprend aussi comment la voix s'inscrit dans ce processus ou cet art (cette technique) de conter ou de chanter des histoires, et comment c'est justement ce couple corps-voix qui donne naissance à l'oralité à travers la performance publique.

D'autre part, ce sont précisément les variations entre les différentes performances qui créent la mémoire orale et l'espace où se lira l'historicité d'une tradition orale. C'est pourquoi on ne peut éviter de s'interroger sur le sens de la mise en cassettes, sorte de "désincarnation » du poème et du poète. Ce dernier reste absent physiquement même si sa voix est bel et bien là, à portée d'oreille. Logé non plus dans la mémoire (collective) d'un auditoire à un moment donné, mais enfermé à perpétuité sur bandes avec son récit - version certes plus "orale» que les tentatives de restitution d'épisodes par écrit - le conteur des cassettes d'Abnûdi engendre à son tour ce qui pourrait passer pour des formes renouvelée du folklore. Or, ne s'agit-il pas en fait de phénomènes d'ordre plutôt folklorique, exportables dans un premier temps à l'intérieur des frontières du monde arabe dont c'est somme toute l'héritage culturel, et in fine dans le microcosme des touristes en mal « d'authentiques traditions »?

ContesLe rythme, une structure

Pour bien réciter, pour bien chanter, il s'agit tout d'abord de mémoriser un rythme. A l'instar de l'apprentissage de la musique orientale, on apprend tout d'abord à taper le rythme sur le târ (tambourin) pour ensuite le faire entrer dans les vers, dans la scansion par exemple, dans l'harmonie rythmique du vers en tout cas. Il me parait tout à fait important de le souligner, car les mots employés pour dire la "parole» n'ont finalement plus beaucoup d'importance. On trouve très couramment, dans la poésie populaire dialectale, des vers qui « sonnent », qui sont «justes » et «beaux » selon des critères bien définis, et qui n'informent en fait que peu sur un sujet précis. On entre dès lors dans un langage plus poétique que descriptif ou logique. La poésie orale procède en effet d'une stratégie de suggestion.

On sait d'autre part que l'arabe ne possède pas le verbe être, ce qui est par définition une des particularités des langues sémitiques. De ce fait, la langue arabe a recours à une série de morphèmes capables de le suggérer et de le faire entendre. La copule kâna (devenir) - et sa version égyptienne dialectale baqa (demeurer) - peut jouer ce rôle, comme le nom d'action (masdar), l'adverbe ou encore certaines prépositions augmentées de pronoms affixes. Il ne faut alors point s'étonner de trouver à l'intérieur même des contes et autres histoires inventées par les poètes des quantités de couplets formés de suite de mois (noms, adjectifs, prépositions, adverbes) qu'il va falloir agencer. L'auditeur reçoit en quelque sorte un «matériau » poétique avec lequel il va devoir construire un (ou plusieurs) sens à la parole émise. Le poète qui connaît son métier se situe exactement à cet endroit : c'est cet espace qu'il investit pour montrer son savoir-faire. C'est là qu'il joue avec et sur les mots, qu'il crée des « promiscuités » de mots, qu'il construit et détruit les racines de mots de même famille, et bien sûr c'est là qu'il donne à entendre son savoir-dire tissant les sonorités, les assonances et les rimes. En ce sens, on pourrait dire que ce qui différencie la prose - elle aussi est soumise à des règles strictes - de la poésie est que cette dernière possède indubitablement une 
musicalité différente produite et par le rythme et par la rime. Le Coran n'est-il pas écrit en prose rythmée et rimée ${ }^{8}$ ?

La rime, une trace

31 Si l'on se penche sur les travaux des critiques arabes de l'époque classique, on s'aperçoit que tous traitent le problème de la rime d'une manière différente. Il y a en fait plusieurs écoles: ceux qui considèrent que le vers entier doit rimer, ceux qui pensent que le dernier mot en entier doit rimer, ceux qui parlent de rimes en relation avec le nombre de syllabes. Le mot utilisé, qâfiya, est lui-même ambigu, puisqu'il signifie littéralement "être derrière quelqu'un ou quelque chose, suivre sa trace ", ce qui ouvre effectivement la porte à de nombreuses interprétations'. Quoi qu'il en soit, tous les auteurs sont d'accord pour affirmer que les vers d'un poème doivent se terminer par le même groupe de phonèmes, appelé rawî (racine qui signifie abreuver, irriguer et aussi conter, relater).

32 En outre il est absolument interdit d'utiliser plus d'une fois dans un même poème le même signifiant avec le même signifié : autrement dit, on ne peut utiliser deux fois le même mot final dans le même sens. C'est ainsi que, traditionnellement, on place un poète parmi les meilleurs même s'il n'a réussi qu'un seul vers, en raison justement de ces obligations de rythme et de rime auxquelles s'ajoutent les contraintes lexicales et grammaticales.

Dans le conte en dialectal, les règles de la métrique de la langue classique sont à respecter, mais peuvent en quelque sorte être aménagées pour que l'auditoire se reconnaisse d'emblée dans le langage tenu. Ainsi alternent des tournures extrêmement classiques - parfois même coraniques sans être forcément religieuses - et des phrases du plus pur dialectal « régional ». Souvent, il suffit de s'éloigner de quelques kilomètres $\mathrm{du}$ lieu de la récitation du conte pour que certains mots ne soient plus compris. $A$ fortiori lorsqu'on traverse le fleuve. La manière de jouer des ellipses sur la rive orientale n'a rien de commun avec ce qui se pratique sur la rive occidentale. Je l'ai souvent constaté : à Gourna (ouest) on ne comprend pas les dialectalismes de Karnak (est).

Le poète qui sait chanter et conter doit ainsi s'efforcer de frotter les mots les uns contre les autres, de les faire sonner et rimer et, pour peu qu'il ait du talent et qu'il ait le goût du (bon) mot, il se doit de jouer avec ceux-ci et surtout de jouer sur le rapport qu'ils entretiennent entre le signifiant et le signifié. La véritable force du poème se situe dans la puissance de suggestion qui surgit des licences poétiques.

Ainsi, à l'écoute d'un conte, l'auditeur se surprend à être emmené dans une tout autre histoire. Il peut également se perdre en route du fait des nombreux coq-à-l'âne dont le conte est truffé. Cette technique est très fréquente, dans la geste hilalienne comme dans les Mille et Une Nuits, mais on la retrouve aussi dans les poèmes improvisés à partir de la vie des saints, chrétiens ou musulmans. Il ne s'agit pas de passer d'une histoire à une autre ni d'encastrer les histoires les unes dans les autres - phénomènes présents dans les Mille et Une Nuits ou dans ce que l'on appelle communément les contes-cadres - ni enfin d'une simple digression, mais bien du passage d'une idée a une autre n'ayant aucun rapport avec la première.

36 Le poète-conteur a, enfin, la possibilité et la liberté d'inclure dans son poème des éléments humoristiques ou ironiques, voire moqueurs, afin de créer une pointe (tachkîl), tout cela pour pimenter le récit et maintenir les sens en éveil. J'en donnerai un exemple, tiré de l'épisode de 'Aziza et Younes, chanté par 'Awadallah : 
'Azîza gâlet âh, 'Azîza gâlet âh bass el-'eshg ramâni

Sedri tarah tîn u rommâni

(Aziza soupira, Aziza soupira : « Mais l'amour m'a délaissée

Ma poitrine c'est des figues et de la grenade »)

II y a ici un jeu de sons entre ramânî («m'a délaissée ») et rommânî (" grenade »); il y a manifestement un coq-à-l'âne, une ambiguïté sur le sens et sur la forme (on ne sait pas qui parle), assonance, rythme (il faudrait entendre le poème pour mieux le goûter), rime d'un mot entier final (en Haute-Égypte, le «a » est souvent prononcé comme un

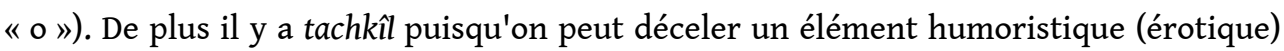
dans cette pointe, qui fait évidemment mouche auprès de l'auditoire. En effet, très souvent dans la littérature arabe (orale et écrite) on compare les seins à des fruits, figues, grenades, melons ou pastèques. En d'autres termes, il faut être très habile pour choisir de faire rimer, en dépit des familles de mots, un verbe suivi d'un pronom affîxe (rama + ni), avec un nom (rommânî).

On voit ainsi avec quelle maîtrise 'Awadallah est plus un nomade de la pensée qu'un simple conteur.

Yâ lêl,yâ 'êni,yâ rabb

Le conte s'organise autour et à l'intérieur de différents cadres. Autour d'une trame, véritable squelette que l'on garnit de chair et que l'on habille. Par ailleurs, il prend forme, c'est-à-dire qu'il ne peut exister que s'il obéit à certaines règles liées non plus au seul environnement (l'auditoire, la tradition), mais bien inhérentes au conte lui-même. Je veux parler des formules emblématiques qui sont les garantes et les gardiennes de la parole à l'intérieur du récit.

40 Le conte s'ouvre généralement sur une incantation, souvent chantée, par laquelle le poète s'en remet au Tout-Puissant pour qu'il l'aide et l'inspire dans son récit. C'est au moyen de celle-ci qu'il lui demande en quelque sorte la permission de raconter. Ces formules liminaires peuvent varier. L'important est qu'elles mentionnent le nom de Dieu, sous une forme ou une autre. Chez les musulmans, on implore Allah ou l'un de ses quatre-vingt dix-neuf attributs et on demande à l'auditoire de saluer le Prophète, que l'on désigne parfois sous d'autres noms (Ahmad, Mahmûd, al-Mustafa par exemple) suivant, semble-t-il, que l'on se réfère à Muhammad sur terre, au ciel ou au paradis.

Chez les coptes, on invoque le Seigneur (yâ rabb) ou la Vierge (yâ 'adra) qui " veilleront " à ce que le conte ne s'éparpille pas et que l'histoire soit conforme aux dogmes religieux, s'il s'agit par exemple de la vie d'un saint.

Pourtant, à l'instar de ce qui se passe dans les chansons, on improvise avant les formules rituelles et religieuses une sorte de poème, qui sonne en fait comme une véritable prière, avec les mots-clés que sont ya lêl (« ô nuit »), ya 'en (« ô œil »), vocables auxquels on ajoute parfois un pronom affixe de première personne du singulier, ya lêli (" ô ma nuit »), ya 'êni (" ô mon œil »). Ces appels généralement plaintifs et chantés sur un mode nostalgique, s'ils n'ont pas réellement de sens - et encore moins en traduction - paraissent indispensables à certains poètes. Ils sont le seuil par lequel on entre dans le poème. On peut les retrouver aussi au milieu de la récitation, soit pour tenir en haleine l'auditoire, soit au contraire pour obtenir de ces deux mots, pendant qu'on les prononce, un court temps qui permet de retrouver l'inspiration et le fil du récit. Ce sont également des sortes de refrains, au même titre que certaines phrases toutes faites qui servent à "coudre " ensemble les différents épisodes. On retrouve d'ailleurs ce système dans l'Odyssée (épithètes homériques) et dans bien d'autres 
littératures, jadis orales, aujourd'hui transcrites, parce qu'il s'agit surtout d'une technique de mémorisation.

Le conte, c'est aussi un moment de plaisir, un moment où la parole, fragile, est offerte à la communauté. Il peut se présenter comme une sorte de scène de séduction entre le poète qui désire dire et la parole qui s'y refuse, et l'on peut parfois assister à un réel envoûtement du poète pour son poème, comme cela arrive aussi chez les musiciens, puisque les uns comme les autres sont les premiers possédés par leur propre " enthousiasme " pour reprendre le mot de R. Arnaldez $z^{12}$. Le conteur peut aussi, à ce moment-là, alterner entre poème chanté et parole récitée, pour changer le rythme intérieur du récit, et ne pas succomber à sa parole. Mais aussi, plus simplement, pour faire avancer l'histoire proprement dite. Il peut en outre répéter une phrase ou un vers, voire un mot, $d u$ fait de son absence réelle de "programme » et pour autant que cela entre dans le cadre du répertoire. En somme, si la phrase est belle, pourquoi s'en priver? A l'instar de la musique, la poésie ne procède pas par développement dramatique, mais par touches légères et brèves qui provoquent le hâl ("état d'être ») bien connu des poètes, des musiciens et des mystiques. Mais revenons sur ces deux formules liminaires. L'une mentionne la nuit, en appelle à la nuit, preuve s'il en est que l'on conte (et chante) de nuit. Parce que l'on travaille de jour, mais peut-être aussi parce que c'est à ce moment-là que les enfants sont couchés ${ }^{14}$. Certains contes, m'a-t-on précisé à Louqsor, ne doivent pas parvenir trop tôt aux oreilles des petits en raison de leurs nombreux sous-entendus. Peut-être aussi les contes doivent-ils être récités de nuit, parce qu'ils renferment une certaine obscurité et renvoient à des lieux indéterminés ${ }^{15}$. La seconde formule (ya 'en) se réfère à l'œil. En arabe le mol «œil » a plusieurs sens : il signifie aussi "la source ", "l'essence». On a vu que conter (rawâ) et abreuver (râwiya) viennent de la même racine, et l'on peut supposer qu'il existe ainsi à l'intérieur de la langue arabe un "terrain de rencontre» de ces deux champs sémantiques, où raconter une histoire serait étancher la soif.

Enfin, mentionner l'œil, c'est aussi empêcher le mauvais œil de s'immiscer dans l'histoire ou dans le conte.

On pourrait d'ailleurs s'interroger sur les symboles et les images que représentent ces deux mots "liminaires » et pourquoi on les accole traditionnellement l'un à l'autre, aussi bien dans les poèmes et les contes que dans les chansons, comme si leur proximité paradoxale - la nuit, l'œil ne voit pas - était nécessaire pour les rendre encore plus équivoques et connotés.

Un « seuil de sortie»

S'il existe un seuil par lequel on pénètre dans le poème ou dans le récit, certaines histoires nous réservent un seuil de sortie, formule permettant de clore définitivement le conte. Il s'agit d'une affirmation plus ou moins courte de l'unicité de Dieu, le conteur pouvant broder et rajouter quelques mots sur la nécessité de casser les idoles, que l'on soit d'ailleurs dans une tradition copte ou dans une tradition musulmane ${ }^{16}$.

Dans ces lieux marqués et hantés par l'héritage culturel des ancêtres pharaoniques, il devient peut-être indispensable de sans cesse réaffirmer sa foi et sa croyance en un Dieu unique...

Le sens

Pour qu'il y ait conte, il faut une " trame » (l'histoire nue) et une " chaîne » (les règles de l'art de conter). Mais il faut encore autre chose, et non des moindres : du ma'nâ, mot 
très difficile à traduire parce qu'il renvoie à plusieurs domaines et à différents niveaux de langues. Il signifie littéralement "sens" ou encore "signification » et entre simultanément en "collision", si l'on peut dire, avec d'autres termes qui appartiennent à la philosophie. En effet, $m a^{\prime}$ nâ traduira tantôt la pensée, tantôt la chose (c'est-à-dire la chose pensée), tantôt le concept voire l'image de l'entendement ${ }^{17}$. Autrement dit, $m a^{\prime}$ nâ représente ce que l'on veut dire, qui est en fait déjà formulé et « dit » clairement dans l'esprit. Une sorte de parole en puissance. Le conte, s'il ne possède pas de $m a^{\prime} n \hat{a}$, est un conte vain. On pourrait ajouter qu'à cause de cela le conteur, qui se doit d'offrir une parole juste et adéquate, possède dans son répertoire une réserve de "morceaux» de phrases ou de vers qui font sens et dans laquelle il puise ce qui lui est nécessaire au bon moment. Rizg, par exemple, lorsqu'il parle de la mort du dragon, tué par Mari Girgis (Saint Georges), n'hésite pas à utiliser l'image de la croix pour décrire les flots de sang, ce qui rajoute du « sens » à l'acte lui-même :

El-damm nâzef w musallab

wel-bahr zâyed w mugallab

(Le sang coula en forme de croix

et le fleuve monta et bascula) tribu et la nostalgie des guerriers, souvent solitaires, dont l'âme souffre d'être loin des êtres aimés et du clan. Ces "valeurs" qui élèvent le caractère de l'auditeur et le rendent vertueux, donc meilleur, sont soit dites en clair, soit codées au moyen de jeux de langage et de mots. Les contes sont par ailleurs truffés de proverbes et de maximes, de paroles bien senties qui se posent comme leçons de morale, mais fournissent un matériau essentiel pour la définition des concepts. Les proverbes sont en effet les 
véhicules de la pensée culturelle, et du fait de leur forme ramassée, la forme la plus accomplie de la parole, la plus libre mais aussi la plus péremptoire.

Le conte offre ainsi un enseignement à plusieurs niveaux et en plusieurs étapes : par le mélange d'arabe classique et de dialectal; grâce aux proverbes qui font office de résumés de l'atmosphère générale distillée tout au long de la geste, de l'histoire ou du chapitre ; enfin par l'entremise de descriptions savantes et directement «imaginées » d'un environnement géographique à portée de main. Car dans la .région de Louqsor, on est frappé d'entendre à travers ces différentes histoires - coptes ou musulmanes, folkloriques ou religieuses - l'exposé minutieux de ce qui fait les particularités de cette région, soit sur le plan social, soit plus simplement sur le plan géographique.

On retrouve en effet dans la plupart des contes telle anecdote qui se rapporte au fleuve ou la mention de tel détail qui s'y réfère. Même si l'histoire se déroule en Arabie Saoudite ou en Tunisie, voire même en plein désert, elle comporte un chapitre sur le fleuve, que l'on décrit toujours comme le Nil en rappelant ses crues et sa mince frange de végétation. Ce fleuve inventé au bord duquel vivent les héros - les bons ou les croyants sur une rive, les méchants ou les mécréants sur l'autre - permet au conteur de décrire un ici et un ailleurs, de créer un monde autre, merveilleux et original. En décryptant le conte de cette manière, on s'aperçoit que dans leur vie quotidienne, les habitants de la région sont très sensibles au fait de provenir d'une rive et non de l'autre. La légère inimitié qui existe réellement dans les actes quotidiens donne en quelque sorte un fondement à ce qui est dit dans les contes et vice versa, le conte permettant lui aussi de mettre en lumière des aspects de la quotidienneté.

On trouve ces éléments en rapport direct avec le fleuve et ses caractéristiques dans l'histoire de Mari Girgis (Saint-Georges) et dans la légende d'Abû-l-Hajjaj par exemple. Le premier, conte copte, narre l'histoire de Saint-Georges qui tue le serpent ${ }^{21}$. Le second semble être de diverses origines : arabe puisque le héros vient du Hidjaz, musulmane dès lors qu'Abû-l-Hajjâj convertit les habitants de Louqsor à l'islam, et pharaonique si l'on se souvient que la reine de Louqsor est une magicienne égyptienne, Tawza ${ }^{22}$. Il raconte notamment comment le héros, au moyen d'une peau de chameau coupée en bandes et réunie bout à bout en une longue ceinture, a réussi à entourer un certain espace où a été bâtie depuis lors la ville de Louqsor, puis comment il s'est octroyé cette terre pour y prêcher l'islam et enfin comment il a fini par épouser cette fameuse reine Tawza.

Dans chacune de ces histoires, que l'on peut entendre en vers ou en prose, on fait référence au Nil. Soit pour «amener» le personnage sur les rivages de Louqsor, soit pour l'en faire partir (le Nil est une voie dans ce cas), ou encore pour décrire l'ambiance de jadis, rythmée par les crues et les saisons et par les différentes récoltes (le Nil devient alors une manne).

Mais si le fleuve peut apparaitre comme une limite, un tracé dans le territoire, il reste aussi, plus simplement, le symbole de la fertilité, de l'imaginaire. Et peut-être faut-il dans un conte s'y référer par principe, et ce d'autant plus lorsqu'on décrit la vie des héros qui sont à l'origine des lieux dans lesquels on continue de vivre. Une manière de s'approprier le passé, non pour vivre à l'ancienne, mais par respect des ancêtres.

Conter

58 On peut se demander quel est aujourd'hui le rôle de ces contes, si riches d'enseignement et si empreints de morale. A l'heure des nombreux programmes de 
télévision diffusés par les chaînes égyptiennes, américaines, françaises, pourquoi est-ce si important et nécessaire de raconter les exploits d'Abou Zêd ou de Mari Girgis ? Même si certains feuilletons ont repris à leur manière les mises en scènes traditionnelles des sentiments chevaleresques, la validité des contes réside dans ce dont les médias modernes sont incapables: remettre en paroles vécues une origine commune, un espace d'imaginaires que l'on partage physiquement avec ses voisins. Les contes permettent encore à ceux qui les écoutent de se sentir ancrés dans une tradition et dans une mémoire. Aussi la télévision, même lorsqu'elle propose des programmes culturels en guise de "vitrine des traditions rurales ", ne contribue-t-elle en rien à la préservation des contes.

On comprend ainsi pourquoi conteurs et poètes attirent les foules lors des foires, même s'ils sont de plus en plus absents de la vie quotidienne. Les contes, grâce à ces rendezvous fixes, continuent d'exister non seulement comme références au passé mais encore comme terreau traditionnel commun, où l'on se retrouve ensemble avec ses valeurs et ses rêves, mais aussi avec son savoir personnel : connaissance de la nature, expérience d'une spécificité géographique, comportement moral et social à respecter. De plus, c'est aussi par leur truchement qu'on peut avoir accès, d'une manière informelle, à la symbolique de la religion, chrétienne ou musulmane.

Les turuq (confréries) n'ont pas à proprement parler de contes ou d'histoires légendaires, bien qu'elles aient toutes une histoire. Elles possèdent par contre des différences notoires en ce qui concerne leurs rites et, cela peut nous intéresser ici, leur manière de louer Dieu, d'évoquer ses noms ou d'invoquer ses attributs sur le mode d'incantations poétiques. Ces dernières favorisent les séances de dhikr, moments intenses des confréries où des formules coraniques, intériorisées au moyen de répétitions rythmiques, font office de prière, accompagnées parfois de mouvements et de gestes, voire de techniques respiratoires particulières.

Un patrimoine culturel commun

61 II convient de revenir sur quelques points concernant les contes proprement dits, une fois le décor général posé. Une des caractéristiques de ces contes populaires, au sens de " pour le peuple ", c'est leur coexistence entre versions écrites et versions orales, voire semi-orales comme peuvent l'être les cassettes enregistrées d'Abnûdi, qui constituent à leur tour un matériau pour d'autres variantes et ainsi renouent sous une autre forme avec ce qui constitue la mémoire orale. Ces allers et retours entre l'écrit et l'oral sont, en ce qui concerne la geste hilalienne, tout à fait significatifs de la situation de la tradition orale dans la région de Louqsor, et peut-être aussi le signe d'un intérêt pour ce qui touche à l'oralité. Car, même si le texte a été arrêté dans l'écrit, même si la geste à été enregistrée sur bandes, voire encore filmée, et qu'ainsi elle peut "exister » n'importe où et à n'importe quel moment, les habitants de Louqsor, pendant le festival d'Abû-l-Hajjâj, continuent d'aller écouter ces mêmes épisodes sur la place publique.

Ce phénomène répond à la nécessité de revivifier périodiquement la convivialité traditionnelle parce qu'elle constitue l'expression rituelle de l'identité à travers la mémoire collective. Celle-ci permet aux musulmans d'écouter les miracles de Mari Girgis, et aux coptes de se sentir concernés par les exploits des Hilâl ou ceux d'Abû-lHajjâj, puisque ce qui est dit dans les contes "colle » aux différents aspects de la vie quotidienne et restaure, au-delà des différences religieuses et sociales, une certaine " égyptianité ». On peut d'ailleurs remarquer que dans les contes chrétiens de la région, lorsqu'il est fait mention de persécutions, il s'agit toujours de celles commises par les 
Romains ou les Byzantins et qu'il n'est jamais question d'animosité entre musulmans et coptes: au contraire ils apparaissent toujours solidaires entre eux parce que monothéistes.

63 En outre, une figure caractéristique de la région telle que Mari Girgis est présente dans le patrimoine culturel tant copte que musulman : en effet, on utilise son nom lors de séances d'exorcisme auxquelles participent les fidèles de l'une ou l'autre religion, dans le couvent de Mari Girgis, près de Louqsor. Ces rituels sont en quelque sorte la contrepartie copte du zâr, séances où l'on entre en contact avec les esprits afin de les amadouer pour finalement s'en débarrasser. Même si ces pratiques ne sont bien vues ni de l'Eglise copte ni des cheikhs orthodoxes de l'islam, elles continuent d'être très fréquentes et très prisées en Haute-Égypte.

Cette communauté de culture s'explique en l'occurrence par le fait que les nombreuses versions qui circulent au sujet des miracles de Mari Girgis plongent leurs racines dans l'héritage pharaonique, surtout en ce qui concerne l'épisode du serpent-dragon qui habite le fleuve: on peut en trouver la filiation dans l'histoire de Seth, qui tue le serpent Apep caché dans les eaux du Nil pour bloquer le passage de la banque de Râ, dieu du soleil.

Ces mythes et ces légendes permettant d'expliquer les crues du Nil, la fertilité de la terre et les cycles agraires, exigent que l'on y revienne encore et toujours à travers la succession des civilisations.

Le temps dans les contes

66 Le temps des histoires contées est une notion fluctuante. Les événements qui en forment la trame ou les caractéristiques qui décrivent la personnalité du héros - telles la ruse ou la fourberie considérées comme preuve d'intelligence - demeurant toujours valides, continuent d'exister dans une sorte de temps "hors du temps ». Le conteur devient ainsi le transmetteur de l'histoire et le témoin de son propre récit. Cela est très caractéristique du conte traditionnel, comme le montrent les nombreuses études comparatives de ces vingt dernières années, mais relève aussi, me semble-t-il, d'une des spécificités de la langue arabe: la grammaire classique ne conçoit pas les temps verbaux comme des états mais comme des aspects. Aussi le temps n'est-il pas une «durée » continue mais, comme le dit L. Massignon ${ }^{23}$, « une constellation, une «voie lactée " d'instants». Le conteur navigue en fait dans un espace plutôt qu'il ne se soumet à des lois temporelles. C'est pourquoi le déroulement chronologique de l'intrigue n'a pas d'importance. Ce qu'il faut à tout prix, on l'a vu, c'est du ma'nâ pour créer des vers "qui conviennent » (elle tenfa') comme le rappelle 'Awadallah.

Cette réalité du conte est vérifiée a contrario par la transcription, laquelle, en s'inscrivant dans la durée, lui-fait perdre son intemporalité. L'écrit met définitivement le conte à la portée de tous, à l'inverse du discours oral qui implique, lui, la présence simultanée, dans le temps et le lieu, d'une bouche qui parle et d'une oreille qui entend. Parole, voix, son, silence

68 La poésie dialectale est difficile à comprendre et à improviser. C'est pourquoi les conteurs, tout comme les chanteurs, ont une sorte de répertoire qu'ils affinent et enrichissent au fil du temps et au gré de l'auditoire. Le passage d'une langue rurale et orale à une expression citadine et écrite, voire le passage à une langue orale mais figée sur bande magnétique, est de ce fait assez périlleux. Car comment comprendre les images, forcément paysannes, lorsqu'elles ne font référence à rien de tangible ou de 
connu ? Comment cette parole peut-elle " agir " sur un auditeur et $a$ fortiori un lecteur, souvent seul devant ce matériau transposé ? Comment supporter la voix du conteur, traditionnellement nasillarde en souvenir du timbre de la zammâra (hautbois ou clarinette) ? Comment entendre les sons et le souffle, les silences, les rythmes, les alternances chant-récitation, pour enfin se laisser pénétrer par cette poésie malgré sa " cohérence de fabrication ", comme le dit si bien A. Miquel ? Et finalement, comment accepter l'intrusion d'une certaine oralité, véritable esthétique de la parole, dans le monde de la poésie oratoire aujourd'hui hors de propos si l'on en croit les poètes arabes modernes ${ }^{24}$ ?

Pour esquisser une réponse à ces questions, il est possible de comparer le sort de la musique à celui du conte. Le cas de Sayyed al-Dawi présente un exemple significatif. Ce poète-chanteur recruté par A. al-Abnûdi pour mettre la geste hilalienne sur cassette a beaucoup plus de succès dans sa région d'origine (Haute-Égypte) que sur les bords de la Méditerrannée ou dans le Delta, parce que la langue sa'îdi est vraiment différente de l'arabe standard. Or si l'on en croit l'étude de S. al-Chawân ${ }^{25}$, tel n'est absolument pas le cas des tentatives réussies d'exportation du folklore musical égyptien, qui tend à gagner non seulement les régions les plus éloignées d'Égypte, mais également celles d'autres pays d'Afrique ou du Moyen-Orient.

70 Ce qui se passe avec la musique, traditionnelle ou populaire, ne correspond à rien dans le monde des conteurs. Aujourd'hui, les chanteurs et les musiciens se produisent dans les halls ou les clubs des hôtels de luxe de Louqsor devant un public principalement étranger, tout comme les danseuses et plus récemment les charmeurs de serpent. Mais il me semble improbable que l'on y voie un jour des conteurs. Parce que les conditions nécessaires à l'art de conter ne peuvent être réunies dans ces lieux, parce que les histoires qu'ils chantent parlent d'une mémoire égyptienne commune, qu'elle fasse référence soit aux ancêtres pharaoniques ou arabes, soit à la double tradition musulmane ou chrétienne. Parce qu'il faut connaitre la langue dialectale, les épisodes des gestes et les vies des saints, ou encore comprendre les proverbes et retenir les jeux de mots. Quand bien même les conteurs appartiennent aussi au "folklore " puisqu'ils deviennent eux aussi objets d'études, on peut penser que les poètes de tradition orale dialectale de Haute-Égypte sont bel et bien, pendant les foires du mois de cha'bân, les seuls et vraisemblablement les derniers piliers de l'exercice de la parole improvisée. C'est à ce titre qu'ils font véritablement partie d'un paysage social toujours présent.

« Raconte une belle histoire ou je te tue (...)

72 Et si mourir était justement de dire non à ce principe suprême, de dire non, (...) si mourir était cette négation inouïe, encore indicible : jamais plus de Shéhérazade? »

\section{BIBLIOGRAPHIE}

(les titres des ouvrages cités dans l'article ne sont pas repris ici) 
Basset R., «Les formules dans les contes », Revue des traditions populaires, $17^{\mathrm{e}}$ année, t. XVII, 1902. pp. 233-243.

Cachia P., Popular Narrative Ballads of Modem Egypt, Oxford, 1989.

Canova G., « Hilaliani e zenâta. Considerazioni sulla Sîrat Banî Hilâl ", Quaderni di studi arabi, 1989, $\mathrm{n}^{\circ} 7$, pp. 163-178,

Al-Shamy H., Folktales of Egypt, The University of Chicago Press, Chicago and London, 1980.

Loret V., "Quelques documents relatifs à la littérature et à la musique populaires de Haute-

Égypte ", Mémoires de la Mission du Caire, second fascicule, Paris, 1885, pp. 305-366.

Norris H. T., «The Rediscovery of the Ancient Sagas of the Banu Hilal », BSOAS, 1988, vol, Ll, pp. 462-481,

Spitta bey W., Contes arabes modernes, Leyde et Paris, 1883.

Van Berchem M., « Conte arabe en dialecte égyptien", Journal Asiatique 1889, t. XIV, $8^{\mathrm{e}}$ série, pp. 84-110.

Yassin M. A , « Spoken Arabic Proverbs », BSOAS, 1988, vol. Ll, Part 1, pp. 59-68.

\section{NOTES}

1. La geste hilalienne se divise généralement en trois cycles : le premier narre l'histoire (sîra) des Benî Hilàl dans leur pays, au moment où l'un des deux émirs, Rizk, épouse Khadra, la fille du chérif de la Mecque. Il en aura un fils, le noir Barakât, appelé plus tard Abu Zêd, personnage principal de toute celte geste. Le deuxième cycle traite de la migration (rihla) des Beni Hilâl dans le Nejd. Ces derniers fuient la famine et se font offrir l'hospitalité par Ghânem et Dyâb, chefs de clan. Le troisième cycle, enfin, raconte la migration vers l'ouesl (taghriba) des Benî Hilàl, en l'an 1068, et leurs guerres avec le souverain de Tunis, père de Sa'dâ qui s'éprendra d'un des compagnons de route d'Abû Zêd, En Égypte, Sa'dâ s'appelle 'Aziza, et le compagnon d'Abû Zêd, dont elle s'éprend follement, est l'Hilalien Younes, et non pas Mar'i, contrairement aux autres versions plus connues de cette geste qui circulent dans les pays arabes d'Afrique et d'Asie.

2. J'emprunte l'image à S. Daney.

3. Comme le montre ce proverbe égyptien : Magnûn w 'aqlo târ w massikûlo f-îdo tàr (" II est fou, son esprit s'est envolé, c'est pourquoi on lui a mis un tambourin entre les mains »), le tambourin (târ) étant un des instruments qui accompagnent les conteurs et les chanteurs de rue.

4. Cf. Canova G., «In Egitîo non esistono amatori di rabâba, ma solo musicisti professionali (zingari) » («En Égypte, il n'existe pas de joueurs de rabâba amateurs, mais seulement des musiciens professionnels, tsiganes »), « Il poeta epico nella tradizione araba, note e testimonianze », Quaderni di Studi Arabi, 1,1983, p. 90.

5. G. Canova parle, lui, de niveau « professionnel » pour le châ'ir et de niveau « amateur » pour tous les autres qui racontent des histoires. A. Al-Abnudi, quant à lui, parle de « responsablité » du châ'ir envers la geste et son déroulement, le râwi étant celui qui conte et n'invente pas. Cf. Actes de la première table ronde internationale sur la geste des Beni Hilâl 26-29.6.1980), Tunis, 1989. 
6. Expériences peu communes au quotidien, mais relativement répandues lors des festivals de théâtre (Avignon, Edimbourg) où l'on privilégie justement ce type d'expériences.

7. Cf. Racy A. J. « The key role of the sammi' [auditeur] is underscored by tha Arab tradition of articulating âdâb « manners » or « codes of behaviour », pertaining to music », World of Music, vol. 33(3), 1991.

8. Cf. Bencheikh J. E. : « Le vrai, le seul poète c'est lui [i. e Muhammad] (je tiens certaines sourates du Coran pour des monuments inégalés de la poésie arabe) parce qu'il fonde un langage qui reconstruit l'univers dans une écriture qui travaille le monde, ce que ne faisaient pas les poètes, simples hommes de bien-dire. ", L'Ane $\mathrm{n}^{\circ} 33$, janvier-mars 1988.

9. Pour plus d'informations, cf. Sammoud H., Ghozzi R., « La définition de la poésie dans l'ancienne poétique arabe ", Poétique $\mathrm{n}^{\circ} 38,1979$,

10. On trouvera d'autres exemples dans Slymovics $S$, « The merchant of Art: an Egyptian Hilali Oral Epic. Poet in Performance », Modern Philology, vol. 120 (Berkeley, Los Angeles and London, University of California Press), 1987.

11. II pourrait toutefois s'agir d'une sorte de « faux archaïsme », bâti sur un vocatif mis au cas indirect (terminaison en « $\mathrm{i} »)$ et non au cas sujet (terminaison en « $\mathrm{u} »)$ comme le voudrait la règle en arabe classique.

12. Cf. Arnaldez R., Les puissances de la langue arabe, Le Genre Humain, Paris, 1990.

13. Comme on dit " manière d'être " et " état d'âme ".

14. C'est le cas en Roumanie (cf. Alvarez-Pereyre F.).

15. C'est le cas des proverbes chez les Bambara. Cf. Zahan D., La dialectique du verbe chez les Bambara, Paris, 1963, p. 104.

16. Je l'ai moi-même enregistré à la fin d'une version de Mari Girgis.

17. Cf. Arnaldez R., Grammaire et théologie chez Ibn Hazm de Cordoue, Paris, 1956, p. 52, cité par Loucel H., « L'origine du langage selon les grammairiens arabes », Arabica, 1963-1964.

18. Voir à ce sujet le dernier chapitre de la thèse de Slymovics, op. cit.

19. Jamâl d'ailleurs, lorsque je l'interrogeais sur un mot inconnu que je croyais néanmoins pouvoir comprendre et deviner, me disait souvent cette phrase sybilline "khalliki 'ala-l-ma'nâ » (« reste sur l'idée », « reste dans le sens », ou « poursuis, dans cette direction »?).

20. Cf. Ibn Tabataba (Xe s.) : «II y a des poèmes, qui, lorsqu'on les démolit et qu'on en fait de la prose, ne perdent ni l'excellence de leurs idées, ni la fermeté de leurs mots ", phrase qui résume assez bien ce que ma'nâ veut dire ! (lyâr al-shi'r, l'étalon de la poésie). Le Caire, 1956, cité par Kilito A., Poétique XXXVIII, 1979.

21. On remarquera qu'en arabe, serpent et dragon sont confondus. Cf. Coran, sourate « al-' $a$ 'râf », versets 104-107, lorsque Moïse jette devant Pharaon son bâton qui se transforme en dragon. Le serpent biblique est devenu un dragon coranique, comme le suggère une note dans la traduction du Coran de Chouraqui (cf. L'appel, p. 322, note 7, p. 107).

22. Plusieurs versions circulent au sujet de cette reine, que certains nomment Tharzah (Thérèse ?) et considèrent comme une jeune vierge chrétienne... Cf. Legrain G., Louqsor sans les pharaons, Paris, 1914.

23. Cf. Massignon L, Parole donnée, Paris, 1962, p. 321 et suiv.

24. Cf. Bencheikh J. E., op. cit. 
25. Cf. Alshawan S., "Some Aspects of the Cassette Industry in Egypt », The World of Music 1987, $\mathrm{n}^{\circ} 2$.

INDEX

Mots-clés : conte, arabe (langue), Louxor, musique, poésie, Saï (Haute Égypte) 